\title{
Strategies Endogenesde Gestion des Ressources Naturelles : Cas des Forets Sacrees de la Commune de Tori-Bossito au Benin (Afrique de l'ouest)
}

\author{
Hountondji Müller Oscar, \\ Ajavon Ayi Yves Césaire, \\ Tente A. H. Brice,
}

Laboratoire de Biogéographie et Expertise Environnementale (LABEE), Université d'Abomey-Calavi

Sinsin A. Brice, Laboratoire d'Écologie Appliquée (LEA) Université d'Abomey-Calavi (UAC)

\section{Doi: 10.19044/esj.2019.v15n15p12～URL:http://dx.doi.org/10.19044/esj.2019.v15n15p12}

\section{Résumé}

Les forêts sacrées malgré leur rôle très important dans la vie socioéconomique d'une nation, subissent une nette régression due aux actions anthropiques. La présente recherche est une contribution à une meilleure connaissance de la diversité des forêts sacrées et des stratégies endogènes mises en œuvre pour leur conservation dans la Commune de Tori-Bossito. La démarche méthodologique utilisée s'est articulée autour des enquêtes socio anthropologiques auprès des dignitaires, des conservateurs et des tradithérapeutes dans les localités de la Commune abritant ces forêts sacrées. Par ailleurs, ces forêts sacrées ont été géo référencées au moyen d'un GPS Garmin 60. Les résultats obtenus révèlent 51 forêts sacrées réparties dans les six arrondissements de la commune de Tori-Bossito. Elles couvrent une superficie total de 38,55 ha et subdivisées en 3 catégories à savoir : les forêts fétiches (au nombre de 28), les forêts de sociétés secrètes (au nombre de 21) et les forêts cimetières (au nombre de 02). Dix (10) stratégies endogènes de gestion de ces forêts sacrées dont la sacralisation; les interdits; la délimitation ; l'association des divinités ;... ont été mises en évidences. Ces stratégies sont complémentaires et participent effectivement à la conservation de la biodiversité dans la Commune.

Mots-clés : Bois sacrées, stratégies de conservation, Tori-Bossito, sud Bénin 


\title{
Endogenous Strategies of Natural Resources Management: Case of Sacred Forests of the Tori-Bossito Community in Benin (West Africa)
}

\author{
Hountondji Müller Oscar, \\ Ajavon Ayi Yves Césaire, \\ Tente A. H. Brice,
}

Laboratoire de Biogéographie et Expertise Environnementale (LABEE), Université d'Abomey-Calavi

Sinsin A. Brice, Laboratoire d'Écologie Appliquée (LEA) Université d'Abomey-Calavi (UAC)

\begin{abstract}
Sacred forests constitute not only botanical reservoirs in the same way as the protected areas of the State, but also true means of communication, protection, prevention and conjuration of certain misfortunes. The present study is a contribution to a better knowledge of the diversity of sacred forests and the endogenous strategies implemented for their conservation in the Municipality of Tori-Bossito. The methodological approach used was based on socio-anthropological surveys of dignitaries, curators and traditional healers in the localities of the Commune, which are home to these sacred forests. In addition, the geographical coordinates of these sacred forests have been geo-referenced using a GPS. The results obtained reveal 51 sacred forests distributed in the six districts. They cover a total area of 38.55 ha and are divided into 3 categories, namely: the fetish forests (28 in number), the secret societies forests (21 in number) and the cemetery forests (02 in number). Ten endogenous management strategies for these sacred forests have been identified. These strategies are complementary and effectively contribute to the conservation of biodiversity in the Commune.
\end{abstract}

Keywords: Sacred woods, conservation strategies, Tori-Bossito, southern Benin

\section{Introduction}

Dans les Pays en Voie de Développement notamment ceux d'Afrique dont le Bénin, la gestion des ressources naturelles est devenue une question 
plus préoccupante (Dossa, 2004). Bien que le Bénin ne dispose pas de vastes formations forestières comme d'autres pays ouest-africains tels que le Ghana, la Côte d'Ivoire, le Libéria, le Nigeria, la Guinée (Sinsin \& al., 2003), il comprend cependant des îlots forestiers dont les forêts sacrées (Kokou \& Sokpon, 2006). Ces îlots, représentant des fragments de forêts, constituent des réservoirs de biodiversité et jouent un grand rôle dans la conservation de la faune et de la flore de ce pays du couloir sec du Dahomey (Sinsin \& al., 2003), Ainsi, le Bénin regorge de ressources forestières jadis gérées empiriquement par les institutions traditionnelles. Toutes ces ressources connaissent une dégradation du fait de l'exploitation irrationnelle ou illicite du capital forestier (DGFRN, 2001). Les forêts sacrées constituent la méthode traditionnelle de conservation de la biodiversité (Lukétas, 2003).

Les forêts sacrées constituent non seulement des réservoirs botaniques au même titre que les aires protégées de l'Etat, mais aussi de véritables moyens de communication, de protection, de prévention et de conjuration de certains malheurs (Tenté, 2005). Ensuite, elles incarnent certains faits socioculturels originaux de gestion de l'environnement (Lèguèdè, 2011). Aussi, les sites sacrés naturels représentent un patrimoine qui reste méconnu bien que les critères de définition du patrimoine s'appliquent aussi à des éléments naturels. Ils sont hérités du passé, transmis de génération en génération visant à assurer la permanence des pratiques religieuses et des représentations symboliques. Ils font l'objet d'un attachement collectif et servent d'union entre la communauté des morts et celle des vivants et perpétuent l'identité sociale et culturelle. Le souci de conserver les ressources biologiques et génétiques devrait figurer parmi les principales composantes de la planification de l'utilisation des terres et des stratégies d'aménagement des forêts (FAO, 2010).

La conservation des formations forestières est aujourd'hui le premier souci des environnementalistes. Malheureusement, les politiques de reboisement, de protection et de restauration des domaines boisés, ne comblent pas le vide laissé par l'exploitation qui utilise des moyens de plus en plus performants. En effet, la diversité biologique en particulier floristique est souvent menacée à cause de la croissance démographique. Mais il est remarqué à Tori-Bossito que les ilots forestiers sacrés ont tendance à protéger la biodiversité et à empêcher l'occupation anarchique des sols. La présente recherche a pour objectif de contribuer à une meilleure connaissance de la diversité des types de forêts sacrées et des stratégies endogènes mises en œuvre pour leur conservation. De façon spécifique, il s'agira de cartographier la diversité des forêts sacrées et de faire le point de toutes les stratégies de gestion endogène de la Commune. 


\section{Matériel et méthodes \\ Milieu d'étude}

La Commune de Tori-Bossito est comprise entre $6^{\circ} 25^{\prime}$ et $6^{\circ} 37^{\prime}$ latitude Nord, et entre $2^{\circ} 01^{\prime}$ et $2^{\circ} 17^{\prime}$ longitude Est et se trouve au centre du département de l'Atlantique. Elle est limitée au Nord par la Commune d'Allada, au Sud la Commune de Ouidah, à l'Est par les Communes d'Abomey-Calavi et de Zè, à l'Ouest par la Commune de Kpomassè (figure $1)$.

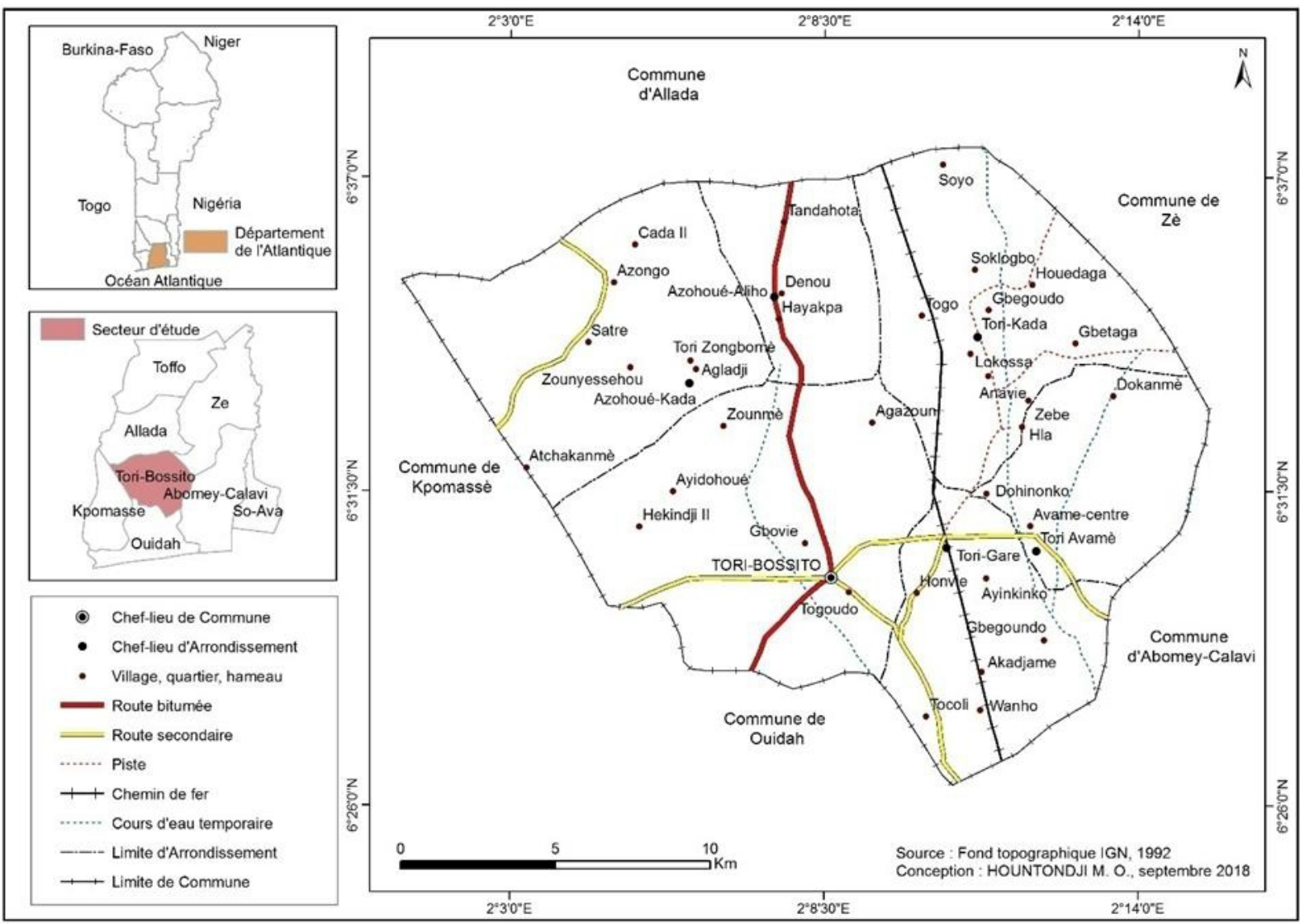

Figure 1 : Situation géographique de la Commune de Tori-Bossito

La Commune jouit d'un climat de type subéquatorial bimodal caractérisé par une alternance de deux saisons pluvieuses et deux saisons sèches (Météo Bénin, 2018).La température moyenne annuelle est de 27,89 ${ }^{\circ} \mathrm{C}$ tandis que la moyenne des hauteurs de pluies enregistrées est de 1442,29 mm d'eau/an (Météo Bénin, 2018). Le couvert végétal naturel est relativement boisé et composé d'îlot de forêt dense, de forêt claire et de savane arborée et arbustive. 


\section{Collecte des données}

L'enquête a couvert tous les villages (35) de la commune de ToriBossito qui abritent les forêts sacrées. Dans chaque village, les entretiens ont été réalisés avec des personnes ressources, notamment les dignitaires des religions endogènes, les dignitaires des religions révélées et les tradithérapeutes. Ces entretiens sont effectués à l'aide de guides d'entretiens et questionnaires d'enquête destinés à chaque cible. Le but visé est de recueillir le maximum d'informations sur les types de forêts sacrées et les stratégies endogènes mises en œuvre pour leurs conservations sur le territoire. $\mathrm{Au}$ cours de cette collecte des données, 65 dignitaires des religions endogènes, 25 dignitaires des religions révélées et 33 tradithérapeutes ont été enquêtés. Ces acteurs de forêts sacrées ont été interviewés à la suite de leur recensement général dans tous les villages. Des séances de focus group ont été tenues par regroupement de villages à proximité. Ainsi, dix focus group ont été organisés afin de mieux trianguler les informations et comprendre les stratégies endogènes de gestion des forêts sacrées.

En marge de la collecte des données socio économiques, des observations participatives sont effectuées avec la prise des coordonnées géographiques de ces forêts au moyen du GPS Garmin 60.

\section{Traitement des données}

Les données collectées ont été dépouillées manuellement et traitées par centre d'intérêt en relation avec le sujet. Ce traitement a permis de réaliser une typologie des forêts sacrées de la Commune, les faits marquant leur sacralisation et les stratégies endogènes de leur conservation. Pour ce qui concerne les coordonnées géographiques, elles ont été projetées sur le fond de carte de la Commune avec le logiciel ArcGIS 10.4 afin de réaliser la carte des forêts sacrées de la Commune de Tori-Bossito.

\section{Résultats et discussion}

\section{Résultats}

\section{Typologie des forêts sacrées}

La figure 2 présente la spatialisation des forêts sacrées dans la Commune de Tori-Bossito. 


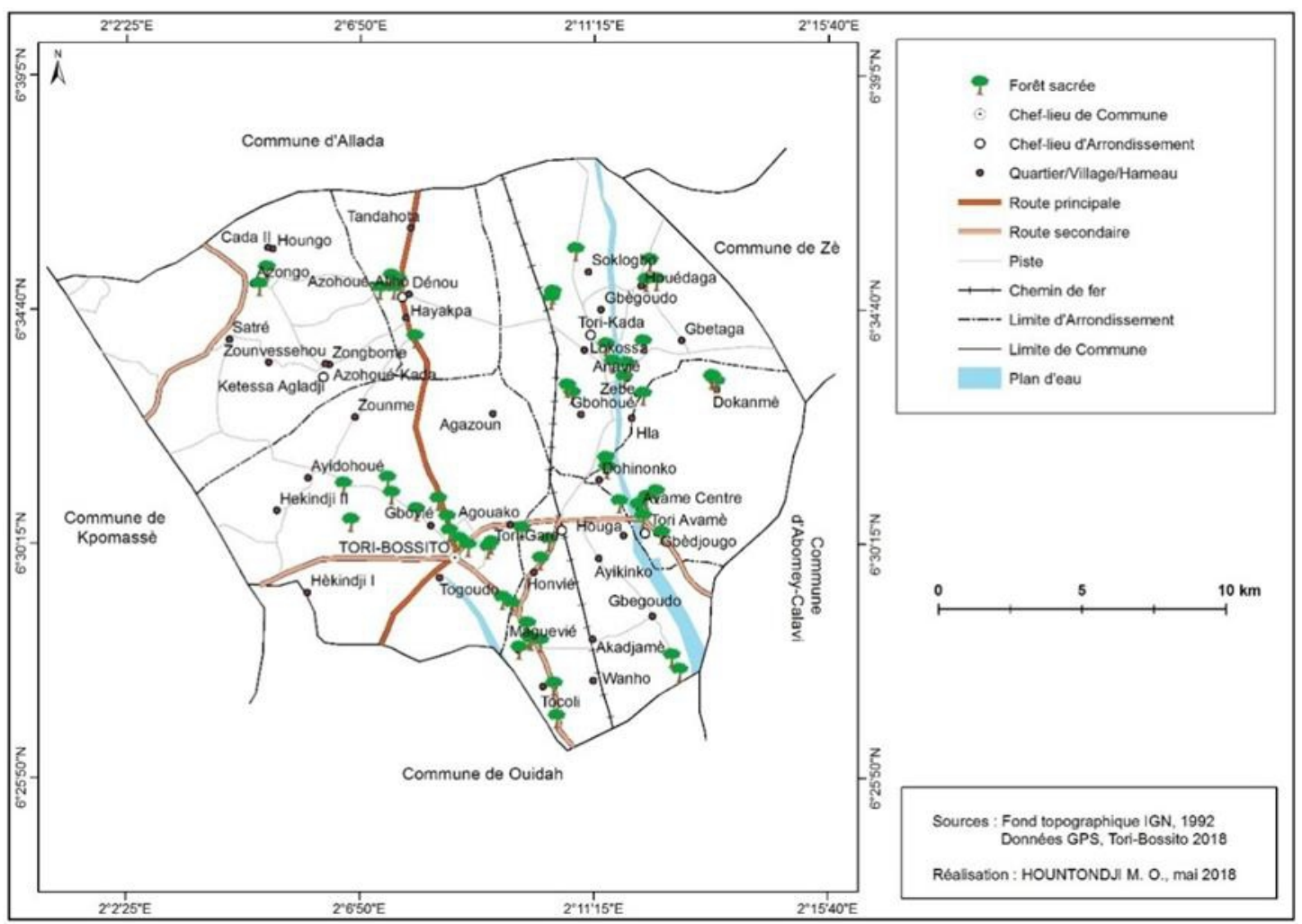

Figure 2 : Spatialisation des forêts sacrées de la Commune de Tori-Bossito

La figure 2, est la répartition spatiale des forêts sacrées de la Commune de Tori-Bossito. Il a été dénombré 51 forêts sacrées réparties dans les six arrondissements (Annexe 1). Ensemble, elles couvrent une superficie de 38,55 ha. Les forêts sacrées sont subdivisées en 3 catégories : forêts fétiches, forêts de sociétés secrètes et forêts cimetières. Il a été dénombré 28 forêts fétiches, 21 forêts de sociétés secrètes et 02 forêts cimetières. Cette nuance a été faite au niveau des forêts sacrées afin d'évaluer dans un premier temps l'importance de la représentation physique de certains fétiches dans les forêts et dans un second temps savoir laquelle de ces trois types de forêts joue un rôle déterminant dans la conservation de la diversité floristique.

\section{Stratégies endogènes de conservation des forêts sacrées}

Tous les dignitaires questionnés soit $100 \%$ ont manifesté le désir de garder pérennes les réserves biologiques. Les raisons fondamentales de cette préoccupation résident en ce que ces forêts regorgent d'espèces médicinales et des divinités. En effet, selon les croyances locales, lesdites divinités jouent un rôle important dans la protection de leurs adeptes, et leur rendent également divers services. Ceci explique l'attachement des populations locales aux endroits consacrés au culte de ces dieux. Les garants de ces forêts 
sacrées sont les rois, les chefs féticheurs, les chefs de terres, les chefs coutumiers et les notables. Autour de ces personnalités qui sont les premiers responsables, se trouvent des collaborateurs qui jouent chacun un rôle bien déterminé dans les cérémonies religieuses et dans le gardiennage. Au total, 10 stratégies de conservation ont été identifiées dans la Commune de ToriBossito.

\section{Sacralisation}

La sacralisation est le fait de décréter une forêt sacrée. C'est tout un processus dont la finalité est d'implanter une ou des divinité (s) dont le nom est souvent donné à la forêt qui l'abrite. Mais certaines informations recueillies sur le terrain font état de ce que parfois lorsque les vieux sentent qu'une forêt court le risque d'être détruite et ainsi de disparaître, cette procédure est enclenchée. En effet, elle a pour finalité l'installation d'une ou des divinités (s) dans un espace boisé qui, après les cérémonies sera décrété sacré. Ainsi, la forêt Kpozoun de Manguèvié s'est vue abriter du jour au lendemain la divinité «Oro » car le KPO (la panthère) qu'elle était sensée abriter n'apparait plus depuis plusieurs générations. Prenant conscience de sa vulnérabilité, les dignitaires y ont installé le «Oro », un fétiche très craint par les plus jeunes de la localité (photo 1).

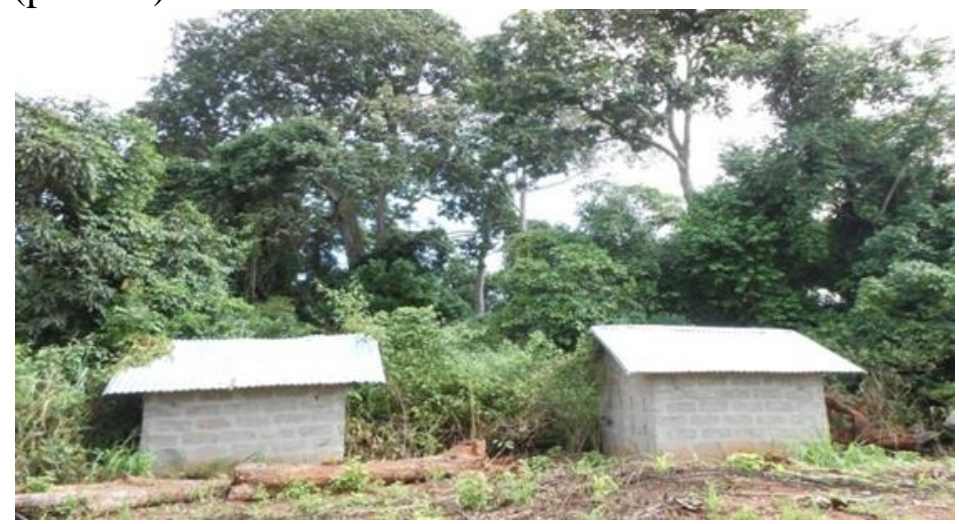

Photo 1 : Deux petites chambres abritant des divinités sur une des limites de la forêt sacrée Zodrèzoun (Manguèvié)

Prise de vue : Hountondji, mai, 2015

La photo 1 montre deux petites chambres contenant des divinités sur une des limites de la forêt sacrée Zodrèzoun (Manguèvié). Ces constructions minuscules remplies de fétiches sont implantées pour arrêter la progression des champs. Au premier plan de l'image on observe le champ de maïs et les deux petites chambres contenant des divinités. En arrière plan se dresse la forêt sacrée Zodrèzoun. Les dignitaires de cette forêt ont construit ces cases pour arrêter le grignotement opéré par le propriétaire du champ voisin à la forêt. 


\section{Association des divinités}

Les résultats de terrain montrent que l'association des divinités est l'un des moyens les plus sûrs dans la commune, non seulement pour protéger et pour conserver les forêts sacrées mais surtout pour leurs superficies. Pour preuve, les forêts les plus étendues de la Commune de Tori-Bossito sont celles qui abritent un grand nombre de divinités.

Les divinités que l'on rencontre souvent dans ces forêts sont :

$\checkmark$ ' 'Gou' dieu du fer, responsable de tout ce qui peut arriver de bien ou de mal dans l'usage des objets métalliques, il intervient auprès de Dieu pour éviter les accidents, les blessures au cours des travaux champêtres ;

$\checkmark$ "Sakpata'" "dieu de la terre', il est considéré comme le responsable des maladies contagieuses en particulier la variole qui provoque les épidémies.

$\checkmark$ 'Dan' dans la tradition locale, c'est le dieu de prospérité par excellence. Il donne de l'argent, il assure l'évolution des activités économiques, la prospérité sinon la richesse à ceux qui le vénèrent.

$\checkmark$ "Tolègba" a une envergure plus étendue et reçoit les sacrifices des individus et des communautés qui l'ont installé.

$\checkmark$ Lègba ou Fa-Lègba fétiche installé à l'entrée des concessions intimement lié à la destinée de chaque individu ayant reçu le "'Fa'.

$\checkmark$ " Oro" symbolise l'ancêtre, il est un fétiche très craint dans la Commune. Malgré son origine Nago ou Yoruba, Il est pratiqué dans tous les arrondissements de la commune de Tori-Bossito.

Ces fétiches à l'exception de "Oro" ont des représentations symboliques ou des temples dans les forêts.

\section{Aménagement des entrées}

Dans plusieurs localités, les gardiens (dignitaires ; adeptes et prêtres) des forêts sacrées nous ont confié que l'entretien ou l'aménagement de l'entrée des forêts sacrées est un signe de vie desdites forêts. En effet, l'entretien de l'entrée d'une forêt sacrée est un moyen efficace pour montrer à tout passant que dans cette forêt se déroulent encore des rites et rituels (photo 2). 


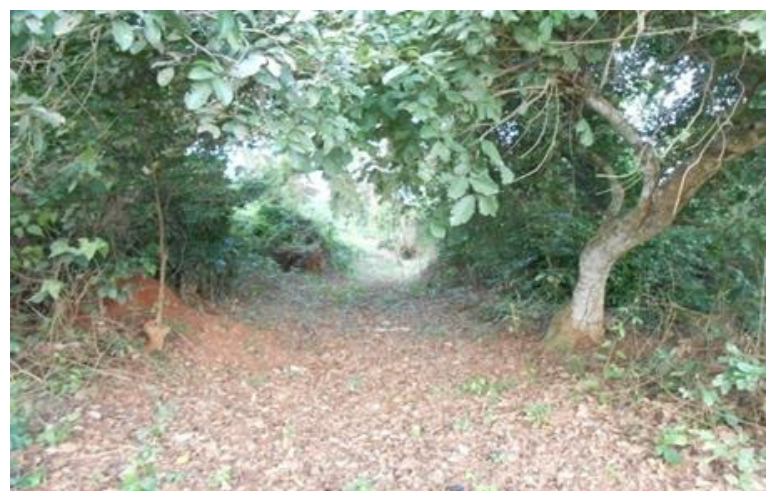

Photo 2 : Entrée aménagée de la forêt sacrée Ahouazinzoun à Agouako Prise de vue : Hountondji, mai 2015

La photo 2 est celle de l'entrée de la forêt sacrée Ahouazinzoun à Agouako. Cette ouverture comme celle d'autres forêts sacrées est nettoyée de façon périodique pour montrer la vitalité de cet espace religieux et l'aspect d'une fréquentation permanente.

\section{Pare-feu}

Certaines populations savent que pour protéger les forêts sacrées contre les feux de végétation, il faut faire des pare-feu (photo 3) surtout pour les forêts situées juste à côté des champs.

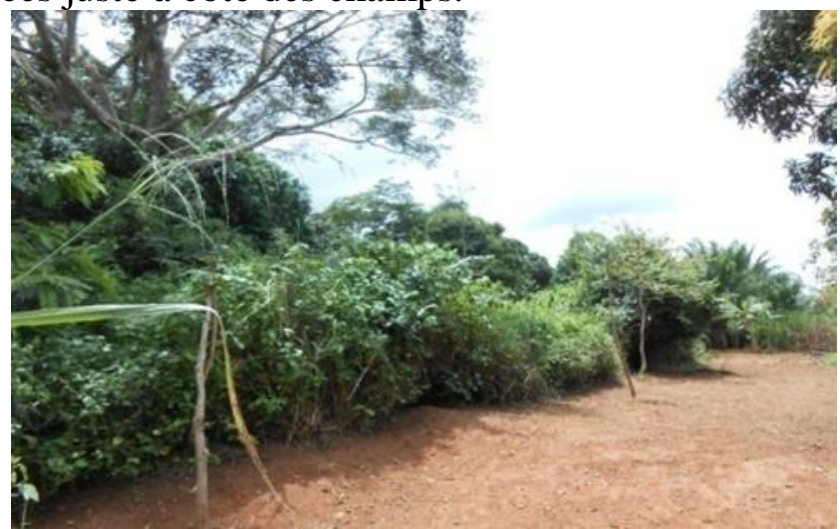

Photo 3 : Pare-feu réalisé autour de la forêt sacrée Orozoun à Dokanmè (Tori-Cada) Prise de vue : Hountondji, mai, 2015

La photo 3 présente de l'est à l'ouest une bande de terre nue (pare feu) aménagée autour de la forêt sacrée Orozoun qui est à l'ouest.

Certaines populations ont compris que les forêts sacrées subissent des dégâts causés par le feu surtout en saison sèche. Alors, ils nettoient, tout autour pour mettre la forêt à l'abri des feux de végétation. 


\section{Délimitation des forêts sacrées}

Plusieurs forêts de la commune ont été délimitées clairement à cause des pressions anthropiques. En effet, les Hommes rognent les forêts pour faire leurs champs ; pour construire leurs habitations ou pour les vendre. Les dignitaires, conscients de cet état de chose procèdent à des délimitations pour freiner ce phénomène (photo 4).

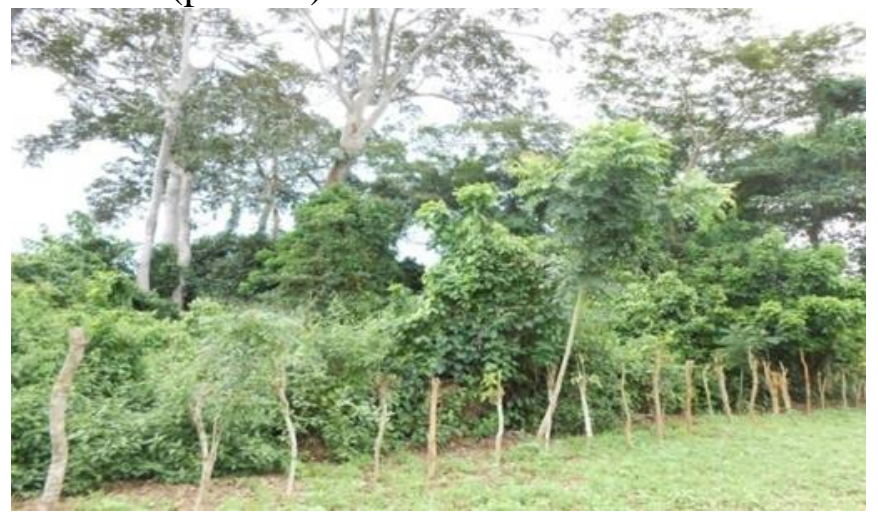

Photo 4 : Délimitation de la forêt sacrée Avazoun dans l'arrondissement de Avamè Prise de vue : Hountondji, mai 2015

La photo 4 présente au premier plan, un champ de niébé. En arrière plan, se trouve la forêt sacrée Avazoun délimitée par les pieds d'hysope (Newbouldia laevis).

Pour arrêter la pression des populations dont les domaines ne cessent de s'étendre au détriment de la forêt sacrée, les dignitaires décident de délimiter clairement cette dernière. La photo est un exemple type de cette initiative. Ces bornes végétales sont placées pour dire "alte" aux « envahisseurs ».

\section{Organisation périodique des cérémonies d'initiation}

Au cour de certaines forêts sacrées de la Commune, les adeptes et les chefs religieux ont aménagé des espaces pour dérouler des spectacles. C'est le cas de Datinzoun à Tori-Cada et de Houèzoun dans Houènonko (ToriBossito). Ce qui est important ici est que même si le spectacle se déroule à l'extérieur sur des places publiques, en amont et en aval, les rites, les rituels et les initiations se déroulent à l'intérieur des forêts qui abritent des couvents. Cela donne tout le cachet secret, sacré de ces cérémonies. La périodicité desdites cérémonies dépend du type de fétiche abrité par la forêt.

Dans les "Orozoun" (forêts abritant la divinité "Oro"), les cérémonies sont régulières et souvent mensuelles ou bimensuelles. Dans les "voudounzoun" (les forêts des adeptes voudoun) où se déroulent les cérémonies d'initiation, les cérémonies sont souvent annuelles ou presque annuelles. C'est d'ailleurs la raison pour laquelle on parle de "houétanoun" 
(le préfixe "houé" signifiant année). Le 10 janvier, étant retenu au Bénin comme le jour de fête des religions endogènes, les prêtes et adeptes trouvent l'occasion idéale pour s'organiser et se mettre en scène.

\section{Inhumations (forêts cimetières)}

La classification des forêts de la Commune a permis d'identifier les forêts cimetières. Ces dernières abritent des "YOHO" ou YOZOUN 'des chambres ou des endroits" cimetières ou les Tori enterrent la tête de leurs morts. Cela fait que les populations autochtones 'les Djakavi' sont particulièrement attachées à ces forêts. Et suivant les témoignages recueillis aux deux palais royaux de la commune et auprès de $100 \%$ des dignitaires interrogés, ces forêts, tout au moins les noyaux centraux ne risquent pas de disparaitre tôt. C'est le cas de Djakazoun à Agonmè (Tori-Bossito), Djaka étant l'ancêtre éponyme de tous les Toli, fondateur du royaume de ToriBossito. C'est également le cas d'Agbokouzoun située à Dokanmè dans ToriCada.

\section{Fermeture des sentiers autour des forêts sacrées}

Les sentiers autour des forêts sacrées rendent vulnérables ces dernières. En effet, lorsqu'une forêt est entourée de passages, elle devient accessible, donc exposée à toute sorte d'exploitation. Les Hommes y prélèvent facilement des essences ; des feuilles ; des racines ; des écorces etc. Conscients de cela, les dignitaires ferment ces passages de manière toute particulière (planche 2).
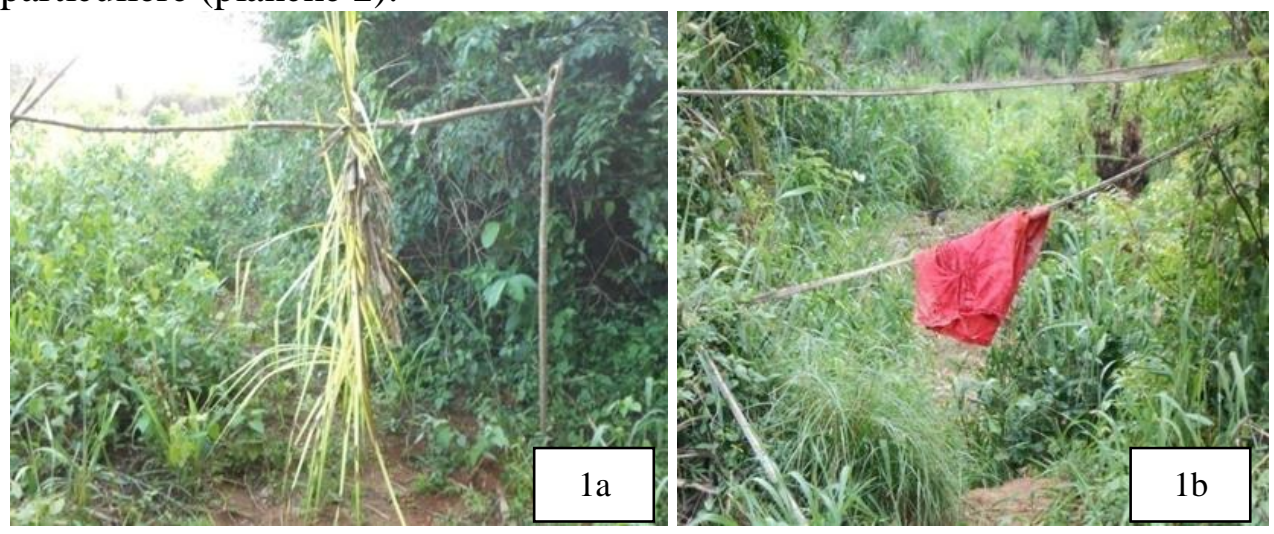

Planche 1 : Fermeture des sentiers autour de forêts Djakazoun à Agonmè (Hounnonko) et Abikouzoun à Bossito I

Prise de vues : Hountondji, mai 2015

Les images $1 \mathrm{a}$ et $1 \mathrm{~b}$ montrent la fermeture traditionnelle des sentiers autour de Djakazoun à Agonmè (Hounnonko) et d'Abikouzoun à Bossito centre. 
Les sentiers exposent la forêt et rendent cette dernière plus vulnérable. A cause de ces ouvertures, les populations peuvent accéder facilement aux forêts pour faire toute sorte de prélèvement. Les éléments utilisés pour fermer sont, dans la tradition locale, lourds de sens. Si le rouge symbolise le danger, les rameaux de palmier signifient que l'offense est répréhensible.

\section{Implantation des plaques d'interdiction}

Lorsque les menaces deviennent de plus en plus pressantes sur les forêts sacrées, les adeptes, les dignitaires sous l'impulsion du palais royal si non du Roi de Tori-Bossito implantent des plaques à des points stratégiques. Ces plaques portent un message interdisant toute exploitation et fait également mention du palais pour effrayer les usagers (photo 7).

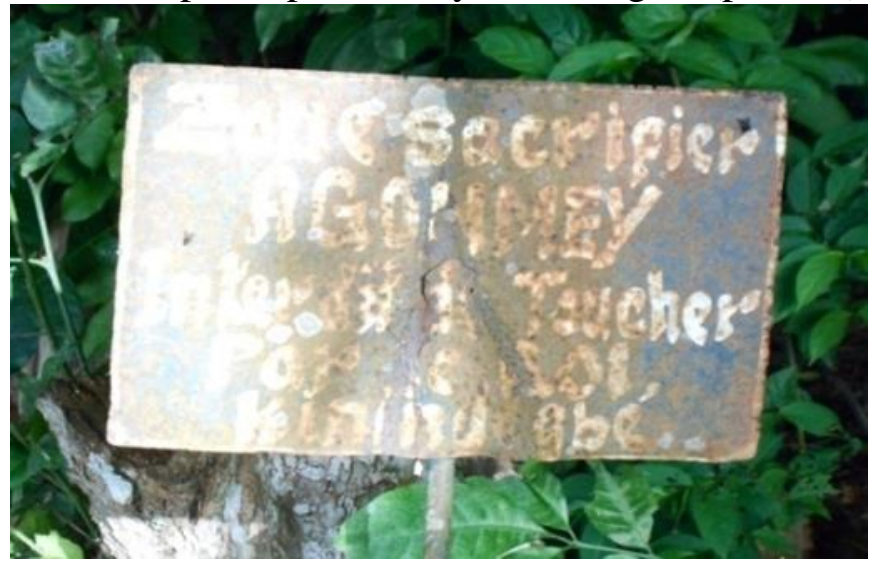

Photo 7 : Plaque d'interdiction de coupe ou d'occupation de la forêt sacrée Djakazoun à Agonmè (Hounnonko)

Prise de vue : Hountondji, mai, 2015

La photo 7 présente la forêt sacrée de Djakazoun qui est une Forêt cimetière. Dans le lotissement de la tranche "A"de la Commune de ToriBossito, elle est maintenue dans son état initial. Mais les populations environnantes exercent sur elle, une forte pression. En 1990, le roi 'Kinidégbé"' de la Commune de Tori-Bossito a pris la décision d'implanter des plaques métalliques portant l'interdiction formelle de toucher à cette forêt.

\section{Interdits}

Certaines populations de la Commune de Tori-Bossito respectent des interdits et des totems en relation avec les ressources végétales. C'est le cas par exemple de la feuille du fromager "Ouman" en langue locale dans laquelle le Tori ou le Djakavi ne doit jamais manger. Du coup, cette feuille est écartée des usages comme emballage dans la commune. Le respect de cet interdit fait que l'arbre qui porte cette feuille est souvent ignoré ou craint (photo 8). 


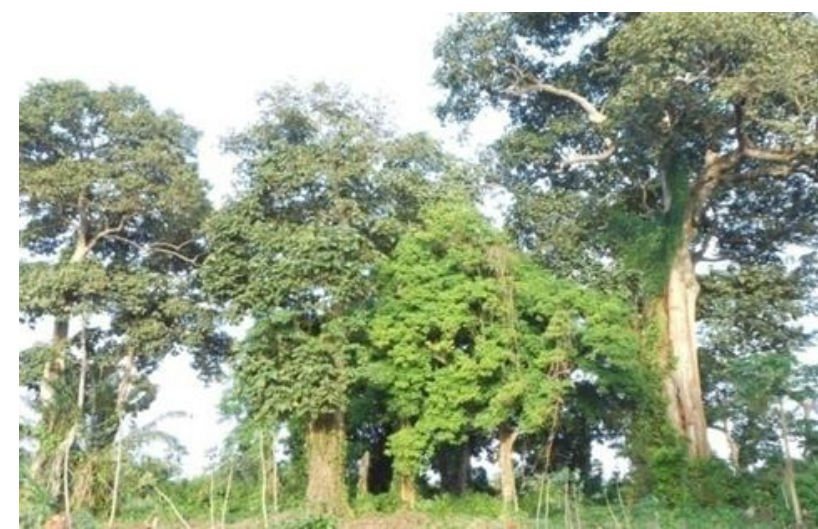

Photo 8: Colas Gigantea de la forêt sacrée Azogbozoun à Manguèvié

Prise de vue : Hountondji, mai 2015

La photo 8 présente la forêt sacrée Azogbozoun à Manguèvié caractérisée essentiellement par le faux colatier (Colas gigantea).

Dans le milieu d'étude, certains dignitaires ont compris que pour protéger certaines ressources naturelles contre les Hommes, il faut les frapper du sceau d'interdiction. C'est le cas du Colas gigantéa dans la Commune de Tori-Bossito dont l'usage des feuilles est formellement interdit à tous les "DJAKAVI", (descendants du roi 'Djaka', roi fondateur du royaume de Tori-Bossito). Du coup, les populations s'éloignent complètement de cette essence. Ces interdits diffèrent d'une forêt à une autre, d'une divinité à une autre, d'une localité à une autre.

\section{Discussion}

\section{Typologie des forêts sacrées}

En rappel, l'identification du nombre des forêts sacrées de toute la Commune a été faite et géo référencée. Elle a permis de recenser 51 forêts sacrées, réparties sur une superficie de 38,55 ha. Les forêts sacrées sont subdivisées en 3 catégories : forêts fétiches, forêts de sociétés secrètes et les forêts cimetières. Il a été dénombré 28 forêts fétiches, 21 forêts de sociétés secrètes et 02 forêts cimetières. Cette nuance a été faite au niveau des forêts sacrées afin d'évaluer dans un premier temps l'importance de la représentation physique de certains fétiches dans les forêts et dans un second temps savoir laquelle de ces trois types de forêts joue un rôle déterminant dans la conservation de la diversité floristique. Cette subdivision des forêts sacrées a été déjà faite par plusieurs autres chercheurs dans bien d'autres études, tant en sciences environnementales que sociales. Hamberger (2008), dans son étude sur les sites sacrés naturels au Sud-est du Togo, a utilisé cette subdivision pour le cadre social des forêts sacrées, leur gestion, leur fonction dans le religieux, et leur potentiel en tant que réservoirs de la biodiversité. Une étude similaire a été réalisée par Sagbo (2012), dans les reliques 
forestières du Bénin. Mais, ce dernier s'est intéressé au volet mystique de ces fétiches qui ont souvent des représentations au sein des forêts et leur impact sur le développement socio-économique du pays. Pour Sokpon et Agbo (1999), les forêts sacrées peuvent être classées en forêts fétiches, en forêts communautaires et forêts-cimetières. Ali (2014), parle des forêts sacrées et des forêts communautaires. Dans la Commune de Tori-Bossito, il a été observé une prédominance des forêts fétiches (57\%). C'est dire tout simplement que la population de la Commune de Tori-Bossito préfère voir le dieu avant de croire. Ce constat dépend aussi du dieu qui incarne cette forêt, car certains dieux ont, depuis leur existence, des représentations dans les lieux à eux réservés. Le nombre de forêts sacrées varie d'un arrondissement à un autre. Cette étude a permis d'actualiser le répertoire des forêts sacrées configuré au niveau de la mairie.

\section{Stratégie endogène de gestion des forêts sacrées}

Le mode de gestion des forêts sacrées est basé sur l'approche traditionnelle qui consiste à implanter des fétiches dans les forêts. Mais, ces forêts peuvent également être régies par des règles élaborées par des sociétés secrètes. Ce mode de gestion des forêts sacrées a été identifié par Ago (2000), sur le plateau Adja. Pour lui, deux facteurs interviennent dans la gestion des forêts sacrées: la nécessité de se cacher et de protéger les divinités. Sinadouwirou (1997) Boukpessi (2003), Camp (2004) ont observé ces mêmes pratiques respectivement dans les forêts sacrées du département de l'Atlantique au Bénin, du Centre-Togo et dans les bois sacrés de l'aire Nyiglin au Togo. Ce mode de gestion basé sur le surnaturel, est conforme à celui constaté par Gbaguidi (1998), au niveau des forêts sacrées de l'Ouémé, par Juhé-Beaulaton et Roussel (1998), pour les forêts et bois sacrés de l'ancienne côte des esclaves.

Dans la Commune de Tori-Bossito, le mode de gestion de forêts sacrées repose principalement sur le sacré, les interdits et la surveillance collective. L'entrée dans les forêts sacrées n'est plus seulement l'apanage des adeptes, des prêtres et des fidèles. Les scientifiques sont autorisés à y faire des recherches. Il suffit de faire la bonne démarche. C'est ce qui a favorisé l'accès à la plupart des forêts sacrées qui ont fait l'objet de cette étude. Même si l'accès aux forêts sacrées de Lokozoun d'Azohouè-Cada, et les forêts 'Oro' en général n'a pas été facile. Selon Ali (2011), la gestion endogène des forêts sacrées de la Commune de Dangbo constitue un frein pour l'aménagement de ces forêts. Tenté et Gibigaye (2011) et Adjé (2014), ont fait les mêmes remarques dans les forêts sacrées de Zinvié et de Kpanroun au Sud-Bénin et celles de la commune de Bonou. 


\section{Conclusion}

Bien que les forêts sacrées bénéficient d'une protection culturelle et religieuse, elles subissent de nos jours de forte pression, de dégradation liées à la pression démographique et aux besoins socio économiques de plus en plus aiguës (extension des champs de cultures, habitations, exploitation frauduleuse des arbres de valeur, feux de brousse, influence des nouvelles religions...). Les habitats de nombreuses espèces sont dégradés, fragmentés ou ont disparu dans certains cas ; ce qui met en péril la survie de l'ensemble de la diversité biologique. C'est pourquoi il est aussi important de réfléchir sur la dynamique des forêts sacrées du territoire de la Commune de Tori-Bossito.

\section{References:}

1. ADJOSSOU Kossi, 2004, Diversité floristique des forêts riveraines de la zone écologique IV du Togo. Mémoire de DEA biologie de développement, option biologie végétale appliqué, Université de Lomé, $75 \mathrm{p}$.

2. ADOMOU Aristide, 2005, Vegetation patterns and environnemental gradients in Benin: implications for geography and conservation. $\mathrm{PhD}$.Thesis Wageningen University.Wageningen.The Netherlands. ISBN 90-8504-305-5.133p.

3. AGBO Valentin. \& SOKPON Nestor, 1998, Forêts sacrées et patrimoine vital au Bénin $32 \mathrm{p}$.

4. AGBO Valentin, SOKPON Nestor, 1997, Forêts sacrées et patrimoine vital au Bénin, Projet CRDI n ${ }^{\circ}$ 95-8170, FSA-UAC, 200 p.

5. AGO Euvariste, 2000, Sacralisation et niveau de maturation des forêts denses semi-décidues du plateau Adja au Sud-Ouest du Bénin. Thèse d'Ingénieur Agronome, Faculté d'Agronomie, Université d'AbomeyCalavi, 172p.

6. Ali Rachad Kolawolé Foumilayo Mandus, 2011. Conservation de la diversité floristique à travers les pratiques endogènes dans les forêts sacrées et communautaires de la Commune de DANGBO. Mémoire de Maîtrise de géographie, UAC, FLASH, 96p.

7. BOUKPESSI Tchaa, 2010. Les pratiques endogènes de conservation de la biodiversité au Centre-Togo. Thèse de Doctorat unique en Géographie. Université de Lomé 280p.

8. CHABI Roméo Brice Kolawolé, 2016, Cartographie des changements spatio- temporels des formations végétales des aires protégées de Togbin, Pahou et Dogbo au sud du Bénin. Mémoire de Diplôme d'Etudes Approfondies. UAC, 87p.

9. CODJIA Jean Claude, ASSOGBADJO Achille Ephrem, MENSAH Ekué Marius Rodrigue, 2003, Diversité et valorisation au niveau local 
des ressources végétales forestières alimentaires du Bénin.

10. Direction Générale des Forêts et des Ressources Naturelle, 2001, Projet bois de feu phase II : Rapport d'évaluation. Cotonou, Bénin, 30p.

11. FAO, 2010, Foresterie et sécurité alimentaire, FIAT, ROME, 136 p.

12. HAMBERGER Klaus, 2008, Les Sites Sacrés Naturels au Togo de Sud-Est. Cadre Social et Fonction Religieux. Rapport Final IFB, halshs-00346724, version 1-12 Décembre 2008. 52 p.

13. HOUNTONDJI Muller Oscar, 2010, Stratégies de gestion endogène des ressources végétales : Cas des forêts sacrées dans l'Arrondissement de Tori-Gare. Mémoire de Maîtrise de géographie, UAC, FLASH, 90p.

14. HOUNTONDJI Muller Oscar, 2015, Stratégies de gestion endogène des ressources naturelles : cas des forêts sacrées dans la Commune de Tori-Bossito. Mémoire pour l'obtention du Diplôme d'Etudes Approfondies. $98 \mathrm{p}$.

15. JUHE- BEAULATON Dominique. \& ROUSSEL B., 1998. A propos de l'historicité des forêts sacrées de l'ancienne Côte des Esclaves. Dans: Plantes et paysages d'Afrique une histoire à explorer. Edité par : Karthala -C 12 p 353-373 p.

16. KOKOU Kouami, ADJOSSOU Kossi, HAMBERGER Klaus, 2005, Les forêts sacrées de l'aire Ouatchi au Sud-Est du Togo et les contraintes actuelles des modes de gestion locale des ressources forestières, Vertigo, la revue en sciences de l'environnement, vol6, 10p.

17. KOKOU Kouami \& SOKPON Nestor, 2006, Les forêts sacrées du couloir du Dahomey, revue Bois et forêts tropicales n²88 (2) ,15p.

18. LUKETA Henri, 2003. Forêts sacrées et conservation de la biodiversité en Afrique centrale : cas de RDC, Canada, 022S-A3, $186 \mathrm{p}$.

19. Ministère de l'Environnement de l'Habitat et de l'Urbanisme, 2002, Agenda de l'environnement, 202p.

20. SEASTROM Marilyn, 2001, Taux de réponse comme outil de gestion de la qualité des données. Recueils du symposium 2001 de Statistique, Canada. 210 p.

21. SINSIN Brice et $a l .$, 2003, La forêt de la Lama au Bénin : un écosystème menacé sous la loupe. Opuscula Biogéographica Basileensia (Suisse) 3 : 1-32.

22. SINSIN Brice. et al., 2003. La forêt de la Lama au Bénin : un écosystème menacé sous la loupe. Opuscula Biogéographica Basileensia (Suisse) 3 : 1-32. 
23. TENTE Agossou Brice Hugues \& Gibigaye Moussa, 2011. Etat actuel des forêts sacrées du sud Bénin et facteurs explicatifs de leur dégradation : cas des arrondissements de Zinvié et Kpanroun. IMOIRIKISI vol 3, $\mathrm{N}^{\circ} 1,1^{\text {er }}$ Semestre 2011, FLASH-UAC. 53-63.

24. TENTE Brice, 2005, Recherche sur les facteurs de la diversité floristique des versants du massif de l'Atacora : secteur PermaToucountouna (Bénin).Thèse de Doctorat, Université d'AbomeyCalavi, 252 p.

ANNEXE : Récapitulatif de la diversité des forêts sacrées de la Commune de Tori-Bossito, leurs divinités et superficies.

\begin{tabular}{|c|c|c|}
\hline Forêts sacrées et Villages & Divinité (s) présente (s) & Superficie \\
\hline Dohizoun (Dohinonko) & Dohi ; hounvè & 2ha \\
\hline Orozoun et Danzoun (Dokanmè) & Oro ; Dan & 0,30 ha \\
\hline Agbokouzoun (Dokanmè) & Yoho & 0,25 ha \\
\hline Orozoun (Hlabè) & Oro & 0,30 ha \\
\hline Datinzoun (Houngo) & Datin ; & 2ha \\
\hline Orozoun (Sogbé) & Oro & 0,15 ha \\
\hline Lissazoun (Zoungoudo) & Lissa & 0,25 ha \\
\hline Houngbanou (Zoungoudo) & Hounvè ; Lissa & 0,5 ha \\
\hline Vodounzoun (Soclogbo) & Hounvè & 0,20 ha \\
\hline Fazoun et Orozoun (Houngo) & Oro ; Fa & 0,5 ha \\
\hline Sakpatazoun (Tanto) & Sakpata & 0,50 ha \\
\hline Agassouzoun (Tori-Cada Centre) & Agassou & 0,50 ha \\
\hline Orozoun (Lokossa) & Oro & 0,20 ha \\
\hline Lokozoun (Azohouè-Cada Centre) & $\begin{array}{l}\text { Cada loko; Ahouanga ; } \\
\text { Todjisso ; Hounfanloko ; } \\
\text { Avizè; Todan ; Tolègba }\end{array}$ & 6,10ha \\
\hline Hinnouzoun (Azohouè) & Hinnou & 1,5ha \\
\hline Azogbozoun (Azohouè-Aliho Centre) & Azogbo, & 0,5 ha \\
\hline Zounkpa (Azohouè) & $\begin{array}{l}\text { Lègba ; Dan ; Loko ; } \\
\text { Azogbo ;Ayossi }\end{array}$ & 4ha \\
\hline Avazoun (Hounsa) & Ava & 0,5 ha \\
\hline Hêbiossozoun (Azohouè-Aliho) & Hêbiosso & 0,5 ha \\
\hline Orozoun (Avamè centre) & Oro & 0,25 ha \\
\hline Orozoun (Assissrekanmè) & Oro & 0,25 ha \\
\hline Hêbiossozoun (Houngo) & Hêbiosso & 1ha \\
\hline Avazoun (Avamè centre) & Ava & 0,30 ha \\
\hline Orozoun (Tookpa) & Oro & 0,25 ha \\
\hline Dossouzoun (Dossou-somey) & Vodoun & $0,75 \mathrm{ha}$ \\
\hline Orozoun (Dossou-somey) & Oro & 0,30 ha \\
\hline Ahouazinzoun (Agouako) & $\begin{array}{l}\text { Ahouanzin ; Lègba ; } \\
\text { Zangbéto }\end{array}$ & 0,5 ha \\
\hline Ahouazizoun & Ahouazi & 0,75 ha \\
\hline Zonhizoun (Gbovié) & Zonhi ; Oro ; Dan & 1ha \\
\hline Danzoun (Kokahoué) & Dan & 0,25 ha \\
\hline Azizazoun (Kokahoué) & Aziza & 0,5 ha \\
\hline
\end{tabular}




\begin{tabular}{lll}
\hline Ouyèzoun (Gbovié) & Ouyè & $0,5 \mathrm{ha}$ \\
\hline Wlénanzoun (Gbovié) & Wlénan ; Ouyè ; Hessou & $4 \mathrm{ha}$ \\
\hline Houèzoun (Honvié) & Houè & $0,5 \mathrm{ha}$ \\
\hline Danzoun (Hounnonko) & Dan & $0,25 \mathrm{ha}$ \\
\hline Ayossigbazoun (Honvié) & Ayossi ; & $1 \mathrm{ha}$ \\
\hline Abikouzoun (Tori-Bossito Centre) & Abikou & $0,25 \mathrm{ha}$ \\
\hline Azogbozoun (Manguèvié) & Azogbo & $0,15 \mathrm{ha}$ \\
\hline Ayossizoun (Hounnonko) & Ayossi & $0,15 \mathrm{ha}$ \\
\hline Zodrèzoun (Manguèvié) & Zodrè & $1,5 \mathrm{ha}$ \\
\hline Dessrezoun (Tori-Bossito Centre) & Hoho, Dan, & $0,5 \mathrm{ha}$ \\
\hline Djakazoun (Hounnonko) & Hoyo & $1 \mathrm{ha}$ \\
\hline Kpozoun (Manguèvié) & Oro & $0,25 \mathrm{ha}$ \\
\hline Orozoun (Manguèvié) & Oro & $0,20 \mathrm{ha}$ \\
\hline Zodrèzoun (Manguèvié) & Zodrè & $0,50 \mathrm{ha}$ \\
\hline Orozoun (Tori-Gare) & Oro & $0,25 \mathrm{ha}$ \\
\hline Djèssizoun (Honvié) & Djèssi & $1 \mathrm{ha}$ \\
\hline Awlinzoun (Ananvié) & Awlin & $2 \mathrm{ha}$ \\
\hline Orozoun (Bossito centre) & Oro & $0,3 \mathrm{ha}$ \\
\hline Danvodounmenouzoun & Danvodounmenou & $1 / 2 \mathrm{ha}$ \\
\hline
\end{tabular}

Source : Enquête de terrain, avril 2017 